\title{
Triginal Minimally invasive image-guided interventional management of hepatic artery pseudoaneurysms
} Alicle

\author{
Sameer Vyas, Niranjan Khandelwal, Vivek Gupta, Chirag Kamal \\ Ahuja, Ajay Kumar, Naveen Kalra, Mandeep Kang, Mahesh Prakash.
}

\section{ABSTRACT}

Department of Radiodiagnosis, Postgraduate Institute of Medical Education and Research,

Chandigarh, India

\section{Correspondence:}

Dr. Niranjan Khandelwal

Email: khandelwaln@hotmail.com

\begin{abstract}
Background: Hepatic artery pseudoaneurysms (HAPs) are uncommon entities. With the development of interventional techniques, their management has evolved from conventional (surgical) to non-surgical minimally invasive image-guided interventional techniques.

Methods: Fifteen cases of HAPs who had undergone non-surgical interventional management in our department were reviewed. All patients were comprehensively evaluated for demographic information, morphology of pseudoaneurysm, indication for intervention and means of intervention, approach (endovascular or percutaneous), follow up and complications.

Results: Trauma and iatrogenic injury were most common causes of HAPs. Most of the HAPs ( 9 out of 10 in whom long follow up was available) managed with image-guided interventional techniques had favorable outcome.

Conclusion: Minimally invasive image-guided interventional management is the preferred modality for HAPs.
\end{abstract}

KEYWORDS: Angiography, hepatic artery, aneurysm, embolization, CT, Doppler

\section{Introduction}

Visceral artery aneurysms (VAAs) are rare entities. Their incidence ranges from $0.01 \%$ to $2 \%$ in various autopsy and angiography series. Hepatic and splenic aneurysms (true and false) account for the major chunk $(\sim 80 \%)$ of all such cases. ${ }^{1-}$ ${ }^{3}$ Hepatic artery pseudoaneurysms (HAPs) $(80 \%)$ are more common than true aneurysms $(20 \%) .{ }^{4} \mathrm{HAPs}$ result from various inflammatory, traumatic, vasculitic and infective causes. Recent increase in their incidence is due to increased number of percutaneous and endovascular hepatobiliary interventions. ${ }^{5}$ Conventional treatment involved surgical exclusion of the aneurysm from the parent artery with or without arterial reconstruction but was associated with significant morbidity.
The increasing experience with minimally invasive interventional techniques over the past decade has set the stage for its widespread application in various fields especially for treatment of such visceral pseudoaneurysms. Image-guided therapy, both percutaneous and endovascular has resulted in reduced hospital stay, decreased morbidity and better recovery rates.

\section{Methods}

Last two years' interventional radiology records were retrospectively reviewed and 15 cases of HAP who had 
undergone non-surgical interventional management were extracted after approval from the department review and ethical committee. Each patient's demographic information, clinical presentation, laboratory parameters, imaging details and risk factors for any vascular disease were noted from the available data sheets. The site, size and nature of the pseudoaneurysm were noted. The indication for intervention and means of intervention were jointly determined by the managing physician and the interventional radiologist prior to deciding upon the final treatment. The presence of significant symptoms, pseudoaneurysm size and bleeding at the time of presentation were major determinants for planning appropriate management. Endovascular or percutaneous approach was subsequently chosen along with the type of embolization material namely coils, cyanoacrylate glue, thrombin, etc. It was primarily based on the location of the aneurysm (proximal or distal), urgency of the procedure, availability of the material and the patient's purchasing capacity. All patients who required repeat treatment sessions were recorded. Variable duration of follow up was available following embolization, ranging from 3 months to 2 years. Follow up focused on any recurrence or development of intermediate/delayed complications.

\section{Results}

The findings of all 15 HAP patients enrolled in the study are summarized in Table 1. There were 11 males (73\%) and 4 females (27\%) with age range from 10 to 85 years (mean: 46.9 years). None of the patients had history suggestive of any connective tissue or vasculitis predisposing to aneurysm formation. Ten
(67\%) patients had acute presentation in the emergency room with hemoperitoneum, severe post-op drain site blood loss, massive gastrointestinal bleed (hemobilia) and rapid hemodynamic deterioration. The rest $(33 \%)$ presented with insignificant symptoms and hemodynamic compensation but suggestive imaging findings. All had anemia (hemoglobin $<10$ $\mathrm{gm} / \mathrm{dl}$ ), leucocytosis (TLC $>11,000 / \mu \mathrm{L}$ ), elevated liver enzymes with variable bilirubin levels. The cause of HAP was trauma in 10/15 (67\%) (Figure 1); liver biopsy in 2/15 (13\%) (Figure 2); endoscopic retrograde cholangiopancreaticography (ERCP) with stenting (Figure 3); radiofrequency ablation for HCC (Figure 4); and arterial wall inflammation due to cholangitic abscesses (Figure 5) in one patient (7\%). The pseudoaneuryms were detected on ultrasound in $12 / 15$ patients while in the remaining three patients contrast enhanced computed tomography (CECT) was employed for identifying the lesion. CT was done in 12/15 (67\%) patients prior to embolization. All the pseudoaneurysms were intrahepatic in location, ranging from $3 \mathrm{~mm}$ (minimum dimension) to $4.2 \mathrm{~cm}$ (maximum dimension) (mean: $10.75 \mathrm{~mm}$ ) in size. There were either large subcapsular hematomas or significant associated hemoperitoneum in most of these patients. Location of the HAPs included right hepatic artery $(n=9)$, left hepatic artery $(n=4)$ and hepatic artery proper, close to the hilum $(n=2)$.

$10 / 15(67 \%)$ patients underwent endovascular management including coiling $(n=5)$, cyanoacrylate injection $(n=4)$ with or without gelfoam particles and polyvinyl alcohol gelfoam slurry $(n=1)$. Endovascular treatment involved selective catheterization of the parent artery close to the neck and embolizing the arterial segment including the neck with the aim

Table 1: Demographic details including the cause, size and treatment adopted for the hepatic artery pseudoaneurysms

\begin{tabular}{|c|c|c|c|c|c|}
\hline S. No. & $\begin{array}{l}\text { Age } \\
\text { (years) }\end{array}$ & Sex & Cause & $\begin{array}{l}\text { Size of } \\
\text { pseudoaneurysm }\end{array}$ & Treatment \\
\hline 1 & 54 & Male & BTA & $12 \times 9 \times 8 \mathrm{~mm}$ & P/C Thrombin \\
\hline 2 & 23 & Male & BTA & $42 \times 30 \times 25 \mathrm{~mm}$ & $\mathrm{P} / \mathrm{C}$ Thrombin $+\mathrm{P} / \mathrm{C}$ cyanoacrylate \\
\hline 3 & 85 & Male & Inflammationdue to cholangitic abscesses & $9 \times 7 \times 7 \mathrm{~mm}$ & Intraarterial cyanoacrylate \\
\hline 4 & 40 & Male & BTA & $13 \times 9 \times 9 \mathrm{~mm}$ & Intraarterial cyanoacrylate \\
\hline 5 & 10 & Male & BTA & $19 \times 15 \times 12 \mathrm{~mm}$ & $\mathrm{P} / \mathrm{C}$ cyanoacrylate \\
\hline 6 & 25 & Male & Liver biopsy & $13 \times 8 \times 7 \mathrm{~mm}$ & Intraarterial coils \\
\hline 7 & 11 & Male & BTA & $18 \times 12 \times 10 \mathrm{~mm}$ & Intraarterial coils \\
\hline 8 & 40 & Female & Post ERCP CBD stenting & $14 \times 12 \times 8 \mathrm{~mm}$ & Intraarterial coils \\
\hline 9 & 48 & Male & BTA & $6 \times 4 \times 4 \mathrm{~mm}$ & $\mathrm{P} / \mathrm{C}$ cyanoacrylate \\
\hline 10 & 65 & Female & Post RFA & $5 \times 5 \times 4 \mathrm{~mm}$ & Intraarterial PVA \\
\hline 11 & 58 & Female & BTA & $12 \times 7 \times 6 \mathrm{~mm}$ & Intraarterial cyanoacrylate \\
\hline 12 & 50 & Male & Liver biopsy & $8 \times 8 \times 6 \mathrm{~mm}$ & Intraarterial coils \\
\hline 13 & 59 & Male & BTA & $14 \times 10 \times 10$ & Intraarterial coils \\
\hline 14 & 65 & Female & BTA & $7 \times 5 \times 5 \mathrm{~mm}$ & $\mathrm{P} / \mathrm{C}$ cyanoacrylate \\
\hline 15 & 70 & Male & BTA & $12 \times 9 \times 9 \mathrm{~mm}$ & Intraarterial cyanoacrylate \\
\hline
\end{tabular}

BTA: blunt trauma abdomen; CBD: common bile duct; P/C: percutaneous; PVA: polyvinyl alcohol; RFA: radiofrequency ablation 


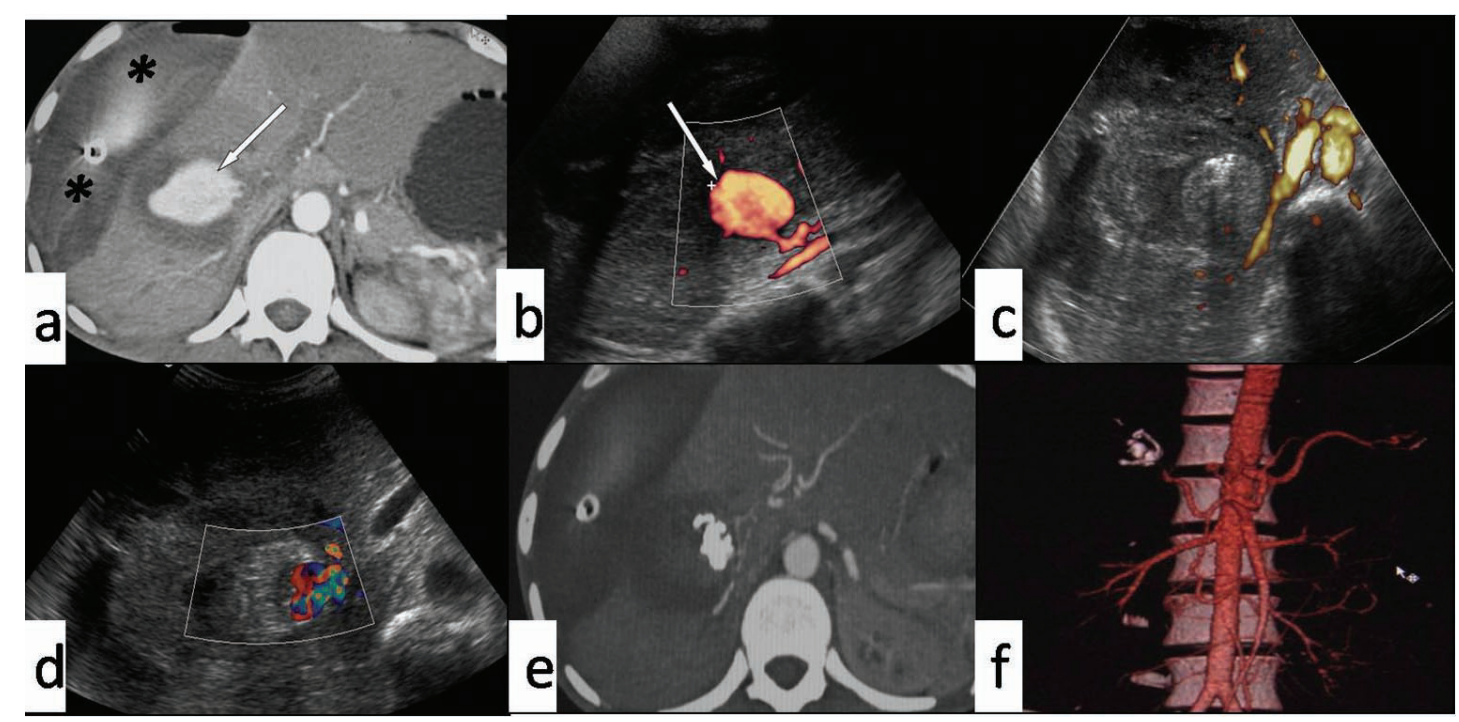

Figure 1: Axial contrast enhanced CT (a) demonstrating pseudoaneurysm in the right hepatic arterial (RHA) territory (arrow). Note the large subcapsular hematoma (asterix) with drain in-situ. Power Doppler USG before (b) and following percutaneous thrombin injection (c) shows echogenic thrombus within the aneurysm sac. Repeat Doppler (d) next day shows partial recanalisation of the aneurysm. Following percutaneous glue injection, there is complete thrombosis of aneurysm (e). VR images of CT following glue injection (f) showing complete thrombosis of the aneurysm. Note the glue cast within the aneurysm sac.

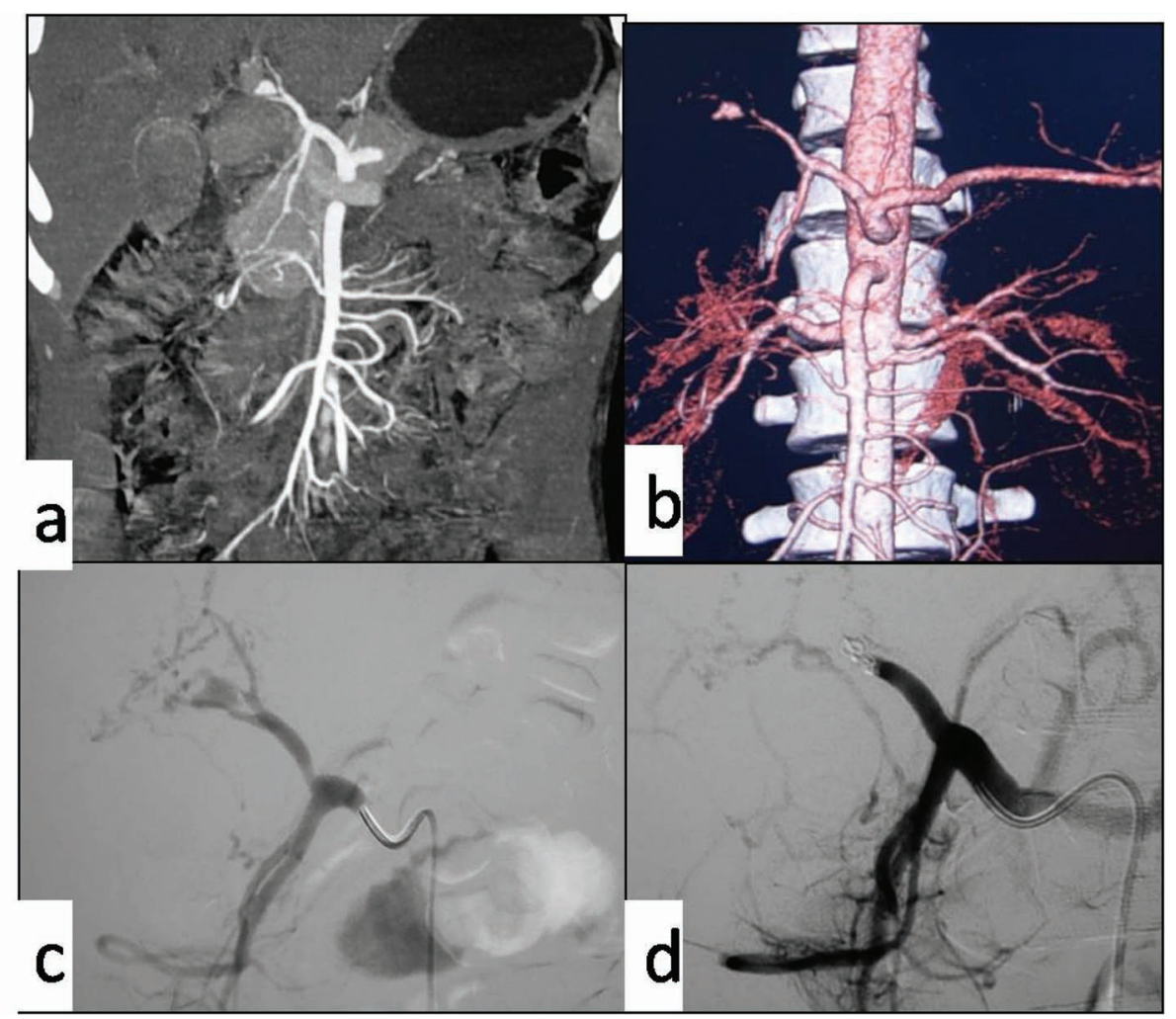

Figure 2: Hepatic artery proper bifurcation pseudoaneurysm in a 25-year-old male following liver biopsy. Coronal MIP (a) volume rendered image (b) of CT angiography demonstrating the pseudoaneurysm at the HA bifurcation. Common hepatic angiogram (c) depicts the narrow neck of the aneurysm. Selective coil embolization of the aneurysm neck was attempted. However, due to markedly short purchase of the parent artery, there was complete occlusion of the hepatic artery proper (d). Follow up CT of the patient showed thrombosis of aneurysm with no hepatic infarction.

of occlusion distal and proximal to it wherever possible. Percutaneous approach under active ultrasound guidance was employed in the remaining 5 patients using glue in 3 (Histoacryl, Braun, United States) and thrombin (Reliseal, Reliance Life
Sciences, India) in 2 patients. One of the latter patients required repeat intervention with percutaneous glue due to partial recanalisation of the pseudoaneurysm. No major procedurerelated complications were noted except for occlusion of the 


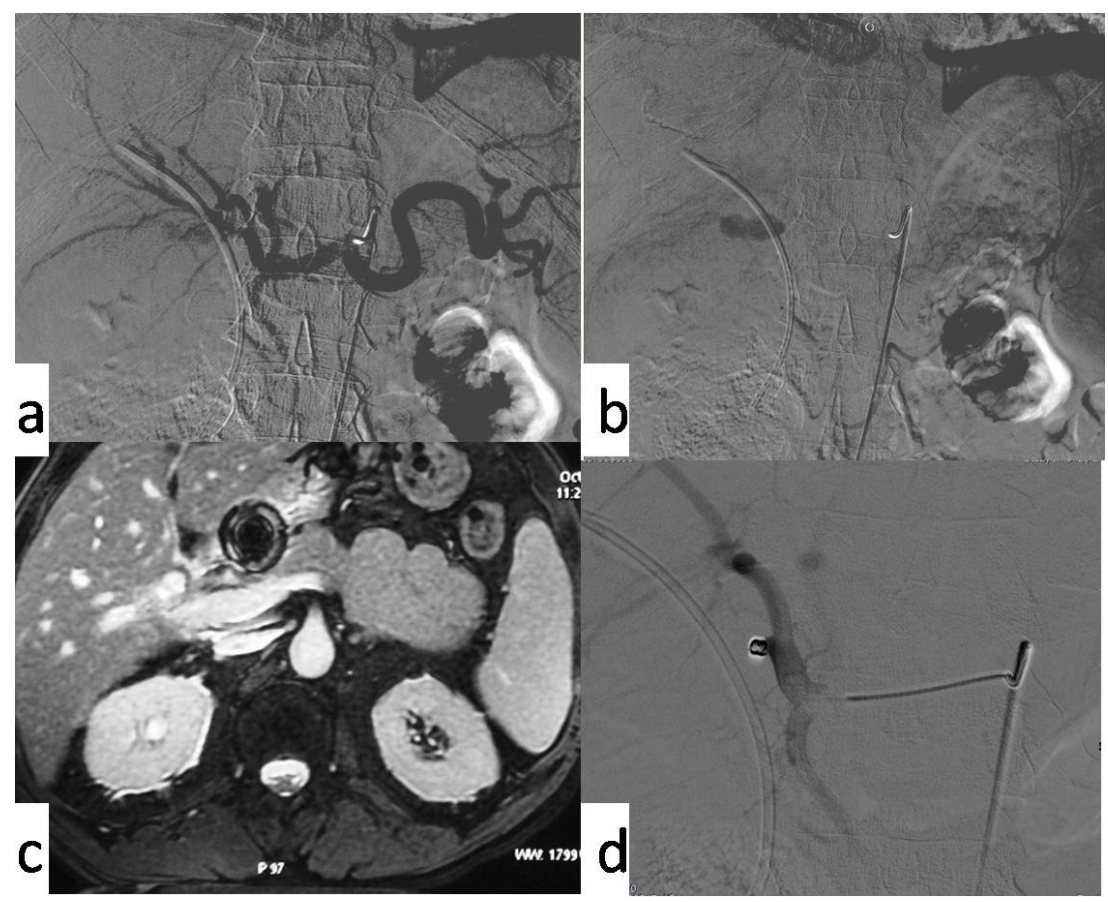

Figure 3: HAP following ERCP guided biliary stenting. Early (a) and delayed (b) phase images of celiac artery angiogram and axial TRUFI MR image (c) depicting the saccular pseudoaneurysm (arrow) arising from the proximal right hepatic artery which was managed by intraarterial coil embolization. Check DSA (d) demonstrating the pseudoaneurysm occlusion.

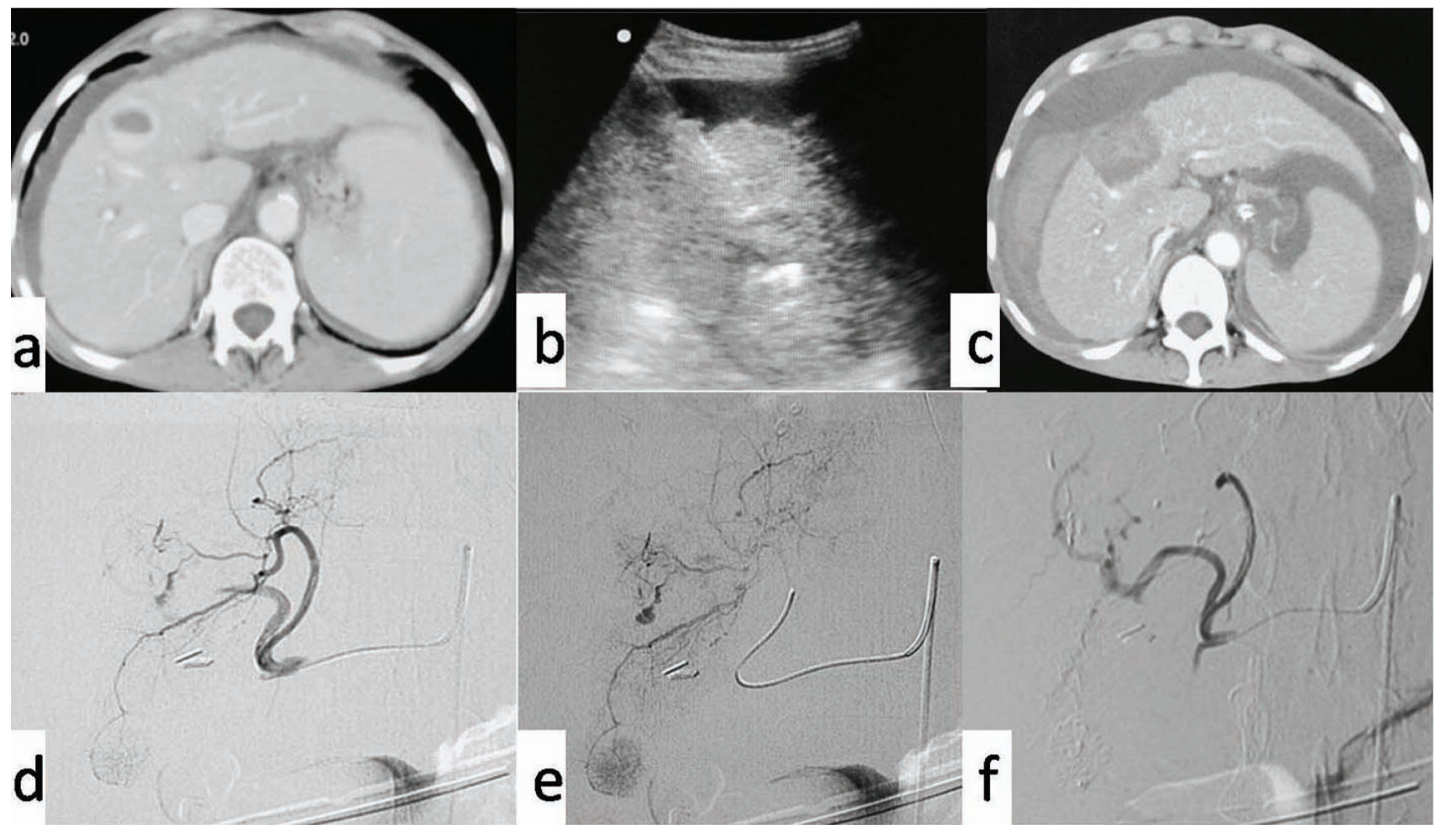

Figure 4: Axial CECT (venous phase) depicting the partially necrotic hepatocellular carcinoma (a) Which was ablated by percutaneous radiofrequency method (b). Check CT (c) showing presence of subcapsular hematoma with hemoperitoneum. DSA showed pseudoaneurysm arising from the bed of the ablated lesion in the early (d) and late arterial (e) phases which was embolised using PVA gelfoam slurry. Check DSA showed non-opacification of the pseudoaneurysm (f). Note made of another small HCC (d, e) which was also chemoembolized with doxorucibin lipiodol mixture during the same sitting.

hepatic artery proper in one patient in whom the arterial stump at the bifurcation from where the pseudoaneurysm was arising was very short. Thus the coil mass had to be deployed in the hepatic artery resulting in complete occlusion of the artery

(Figure 2d). Follow up CT scan done during the following week however, did not show hepatic ischemia indicating that there was adequate compensation by the portal circulation.

The initial treatment was successful in 14 (93\%) which was determined by cessation of flow within the pseudoaneurysm or its successful exclusion from the main circulation by the end 


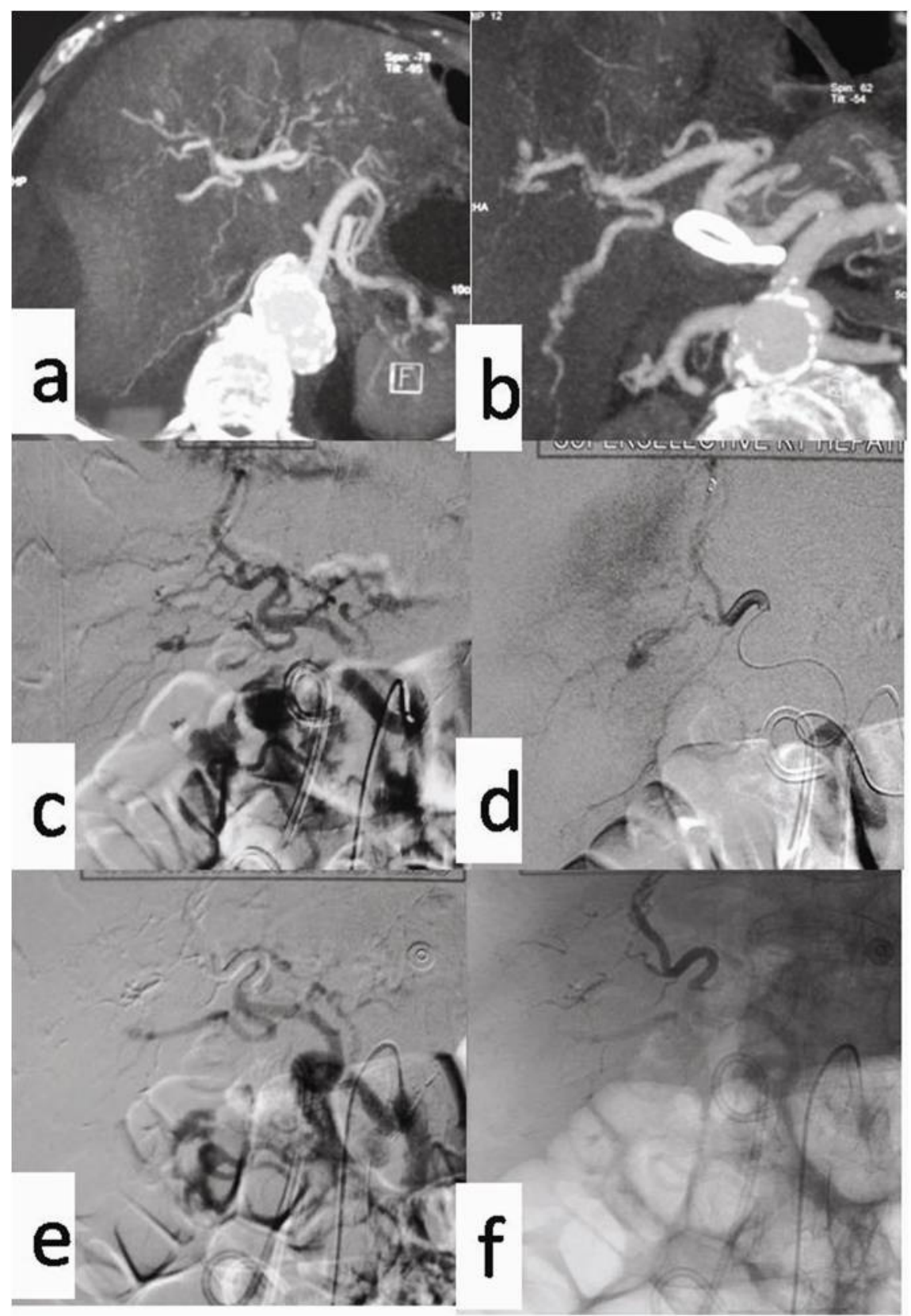

Figure 5: HAP in an 85-year-old man following arterial wall inflammation due to cholangitic abscesses. Axial (a) and coronal (b) MIP reconstructed images of CT angiography showing RHA pseudoaneurysm. Common hepatic (c) and selective RHA (d) angiograms depicting the pseudoaneurysm which was embolised with endovascular cyanoacrylate glue injection. Check DSA (e) showing embolization of the feeding artery with glue cast in-situ (f).

of the procedure. One case which required repeat therapy (Figure 1) was a large pseudoaneurysm in which percutaneous thrombin was injected. However on 24 hour follow up with Doppler ultrasound, there was partial recanalisation which prompted repeat embolization with percutaneous glue. Complete occlusion was thus achieved in this patient and follow up until 6 months was uneventful. One patient (post-ERCP pseudoaneurysm) succumbed to sepsis after 15 days due to choledocholithiasis induced biliary obstruction (Figure 3). He had developed cholangitis earlier which did not resolve due to poor response to antibiotics and old age. Five others were lost to follow up over one month after discharge. Imaging followup with either Doppler ultrasound or CT angiography was available for the remaining 9 patients for variable durations ranging from 3 months to 2 years. No pseudoaneurysm recanalisation was seen in any of these patients on follow-up.

\section{Discussion}

Visceral artery aneurysms (VAAs), though infrequent entities, 
are potentially dangerous if not tackled promptly. Hepatic artery pseudoaneurysms (more common than true aneurysms) are one of the major subgroups of VAAs resulting from various traumatic, inflammatory, vasculitic and infective (mycotic) causes. A recent rise in their incidence during the last decade coincides with the increase in the numbers of percutaneous and endovascular hepatobiliary interventional procedures ${ }^{5}$ as well as laparoscopic cholecystectomy ${ }^{6,7}$ and liver transplantation. ${ }^{8}$ Trauma to the liver remains one of the major causes for the development of hepatic artery pseudoaneurysms. The affected patients present with variable symptoms. Tessier et $\mathrm{al}^{6}$ in their series of 153 HAP patients reported hemobilia as the common presenting symptom (64\%) followed by hematemesis (30\%), hematochezia (14\%) and abdominal pain (20\%). Other unusual presentations depend on the size and location of HAP e.g. pruritis and other obstructive symptoms due to CBD obstruction. Prompt diagnosis of this potentially life threatening condition, especially if the patient is asymptomatic, is crucial for instituting immediate therapy.

Ultrasound with or without CT angiography forms the basis of diagnosis in majority of cases. The use of CT after blunt abdominal trauma or suspected arterial injury after an interventional biliary tract procedure has improved HAP detection. ${ }^{9}$ A distinct advantage of $\mathrm{CT}$ is the concomitant evaluation of other extravascular structures. Magnetic resonance angiography (MRA) is an alternative to CT angiography in patients allergic to iodinated contrast material or having renal insufficiency especially in pregnant and pediatric population. Selective catheter angiography remains the most sensitive modality for detecting HAP. Moreover it can be used simultaneously for diagnosis and treatment of unstable cases minimizing the time to treatment. However, many authors recommend an initial CT scan if HAP is suspected which serves as a roadmap for angioembolization during angiography. ${ }^{10}$

Both intrahepatic and extrahepatic pseudoaneurysms of the hepatic arterial circulation hold high rates of rupture and merit intervention at the time of diagnosis. ${ }^{11}$ Post-traumatic HAPs, despite being asymptomatic should be treated as early as possible given their high risk of rupture and high associated morbidity. ${ }^{1,12,13}$ Earlier, their treatment involved surgical exclusion from the parent artery with or without arterial reconstruction which was fraught with significant morbidity. With the advent of modern imaging and angiographic methods, as well as the increased experience with minimally invasive interventional techniques, these lesions are more often being managed by interventional methods. The indications for HAP intervention have also broadened from symptomatic aneurysms, aneurysms in women of childbearing age and asymptomatic aneurysms over $2 \mathrm{~cm}$ in size in good-risk surgical candidates ${ }^{14}$ to almost all pseudoaneurysms detected on imaging. ${ }^{4}$ Early intervention is the key instead of serial surveillance which is also being followed at our institute except in exceptional circumstances. Our treatment options focus on minimally invasive options as the first-line approach with open surgical intervention reserved for those patients who fail embolization, have coexisting complications or have extremely unsuitable vascular anatomy.

The decision to intervene in the pseudoaneurysms in the present study was reached with a consensus between the primary physician and the surgeon caring for the patient and the interventionalist performing the procedure. This was based on a number of factors including surgeon's/interventionalist's experience, equipment availability, support staff experience and anatomical considerations. The treatment techniques used for each aneurysm was individualized for each patient. Both endovascular and percutaneous techniques were employed depending on the size of the pseudoaneurysm, its location, arterial tortuosity as well as the availability of the necessary hardware (in addition, non-affordability by the patient is a major issue in our setup, with this treatment not covered under insurance schemes). The various embolizing agents used were microcoils, N-butyl cyanoacrylate (NBCA) glue, gel foam particles, PVA particles and thrombin. Others materials which have been successfully used at variable locations by other authors include ethanol, detachable silicone balloons and stents. ${ }^{15-17}$

Ethanol injection has been abandoned due to its sideeffects. Gelfoam has shown success but achieves only temporary hemostasis with very high recanalisation rates. Percutaneous thrombin injection for treatment of visceral, ${ }^{18,19}$ renal $^{20}$ and extremity pseudoaneurysms has been used. However, reperfusion within the pseudoaneurysm is common as was seen in one case in the present study (out of two in which percutaneous thrombin was used). Thromboembolism and allergic reactions are also not uncommon. ${ }^{20} \mathrm{PVA}$ is a very effective material for use with large lumen catheters but does not somehow achieve complete permanent occlusion of the small vessels and pseudoaneurysms. We also used it sparingly in only one patient who had a leaking pseudoaneurysm along with adjacent small vascular trauma induced by the heat generated due to radiofrequency waves. Detachable coils are 
a reasonably good alternative but sometimes these cannot reproduce their predefined shapes in small distal vessels. Cyanoacrylic glue is a permanent and efficient embolizing agent that has been widely used for many years in cranial and extracranial vascular malformations. ${ }^{21}$ Percutaneous injections of cyanoacrylate glue into the visceral, renal and femoral pseudoaneurysms have also been reported rarely. ${ }^{22-24}$ It requires significant precision and operator experience.

Selective arterial embolization is currently considered to be the most appropriate technique for the treatment of pseudoaneurysms, with a success rate of more than $80 \%$. Also it has many advantages over surgery being less traumatic, not requiring general anesthesia, having less parenchymal injury along with shortened hospitalization following the procedure. $67 \%$ patients in our study underwent endovascular treatment with coils used as the most frequent embolization agents (50\% of these patients). Cyanoacrylate glue was used in $40 \%$ patients while in 1 patient ( $10 \%$ of endovascular treated patients), a mixture of gelfoam and polyvinyl alcohol particles was used (Figure 4). Although less common, treatment with endovascular stenting has been reported as an alternative to selective arterial embolization when there is concern about hepatic infarction or vascular recanalisation. ${ }^{17,25}$ The stents are a non-invasive method of excluding the pseudoaneurysm from the parent arterial circulation while retaining hepatic arterial flow. ${ }^{26,27}$ Utmost precision during deployment, high cost, increased risk of stent thrombosis and the need for long term anticoagulation are some of the hindrances to their widespread use. Due to some of these reasons, stents did not find a place in the treatment of any of the patients in the present series.

Ultrasound-guided percutaneous access is an easy technique and was employed in $33 \%$ patients. This technique was used especially in difficult-to-access aneurysms that otherwise would have required open surgical intervention. It is an inexpensive method suitable for patients who cannot afford other options. Both cyanoacrylate glue (3 patients) and thrombin were injected (2 patients) as liquid embolics. Careful details of the pseudoaneurysm milieu and hemodynamics were assessed prior to embolic injection. Narrow necked aneurysms with slow flow were chosen for this approach as introduction of these materials into the arterial circulation can wreak havoc. No complications were encountered with either of these agents.

When embolizing one of the hepatic arteries, intrahepatic translobar collaterals usually establish flow immediately to the ischemic lobe and no infarction occurs. ${ }^{28}$ The risk of ischemia is relatively increased in embolization of the hepatic artery proper. However, with the portal circulation supplying $80 \%$ of blood to the liver, ischemic damage occurs infrequently. It has been reported in some patients though rarely. ${ }^{29,30}$ Patients with underlying liver disease, inadequate collateral flow, or compromised hemodynamic status are prone to liver damage during main artery occlusion. One of our patients in whom the main hepatic artery was occluded during coil embolization did not experience hepatic necrosis as seen in the follow up scans. Apart from hepatic necrosis, coil migration, aneurysm rupture and hepatic abscess are some of the reported complications of endovascular hepatic artery embolization. We did not encounter any of these complications in our series.

It is thus necessary to be aware of all the therapeutic options and to evaluate the advantages and disadvantages of each before adopting a particular method in any given patient. Due to the paucity of studies with large patient cohorts to validate preferred treatment options, the present study hopes to contribute substantially in enhancing the understanding of various treatment modalities; being the largest of its kind and highlighting the special role of image-guided interventional management in such patients.

\section{References}

1. Tulsyan N, Kashyap VS, Greenberg RK, Sarac TP, Clair DG, Pierce G, et al. The endovascular management of visceral artery aneurysms and pseudoaneurysms. J Vasc Surg. 2007;45:276-83.

2. Kasirajan K, Greenberg RK, Clair D, Ouriel K. Endovascular management of visceral artery aneurysm. $J$ Endovasc Ther. $2001 ; \mathbf{8}: 150-5$.

3. Abbas MA, Fowl RJ, Stone WM, Panneton JM, Oldenburg WA, Bower TC, et al. Hepatic artery aneurysm: factors that predict complications. J Vasc Surg. 2003;38:41-5.

4. Fankhauser GT, Stone WM, Naidu SG, Oderich GS, Ricotta JJ, Bjarnason $\mathrm{H}$, et al. The minimally invasive management of visceral artery aneurysms and pseudoaneurysms. J Vasc Surg. 2011;53:966-70.

5. Tessier DJ, Abbas MA, Fowl RJ, Stone WM, Bower TC, McKusick MA, et al. Management of rare mesenteric arterial branch aneurysms. Ann Vasc Surg. 2002;16:586-90.

6. Tessier DJ, Fowl RJ, Stone WM, McKusick MA, Abbas MA, Sarr MG, et al. Iatrogenic hepatic artery pseudoaneurysms: an uncommon complication after hepatic, biliary, and pancreatic procedures. Ann Vasc Surg. 2003;17:663-9.

7. Balsara KP, Dubash C, Shah CR. Pseudoaneurysm of the hepatic artery along with common bile duct injury following laparoscopic cholecystectomy. A report of two cases. Surg Endosc. 1998;12:276-7.

8. Bussenius-Kammerer M, Ott R, Wutke R, Grunewald M, Hohenberger W, Reck T. [Pseudoaneurysm of the hepatic artery- 
a rare complication after orthotopic liver transplantation]. Chirurg. 2001;72:78-81.

9. Shanley CJ, Shah NL, Messina LM. Common splanchnic artery aneurysms: splenic, hepatic, and celiac. Ann Vasc Surg. 1996;10:315-22.

10. Otah E, Cushin BJ, Rozenblit GN, Neff R, Otah KE, Cooperman AM. Visceral artery pseudoaneurysms following pancreatoduodenectomy. Arch Surg. 2002;137:55-9.

11. Wagner WH, Allins AD, Treiman RL, Cohen JL, Foran RF, Levin PM, et al. Ruptured visceral artery aneurysms. Ann Vasc Surg. 1997;11:342-7.

12. Ikeda O, Tamura Y, Nakasone Y, Iryou Y, Yamashita Y. Nonoperative management of unruptured visceral artery aneurysms: treatment by transcatheter coil embolization. $J$ Vasc Surg. 2008;47:1212-9.

13. Pulli R, Dorigo W, Troisi N, Pratesi G, Innocenti AA, Pratesi C. Surgical treatment of visceral artery aneurysms: A 25-year experience. J Vasc Surg. 2008;48:334-42.

14. Saltzberg SS, Maldonado TS, Lamparello PJ, Cayne NS, Nalbandian MM, Rosen RJ, et al. Is endovascular therapy the preferred treatment for all visceral artery aneurysms? Ann Vasc Surg. 2005;19:507-15.

15. Hidalgo F, Narvaez JA, Rene M, Dominguez J, Sancho C, Montanya X. Treatment of hemobilia with selective hepatic artery embolization. J Vasc Interv Radiol. 1995;6:793-8.

16. Yamakado K, Nakatsuka A, Tanaka N, Takano K, Matsumura K, Takeda K. Transcatheter arterial embolization of ruptured pseudoaneurysms with coils and n-butyl cyanoacrylate. J Vasc Interv Radiol. 2000;11:66-72.

17. Venturini M, Angeli E, Salvioni M, De Cobelli F, Trentin C, Carlucci $M$, et al. Hemorrhage from a right hepatic artery pseudoaneurysm: endovascular treatment with a coronary stentgraft. J Endovasc Ther. 2002;9:221-4.

18. Krueger K, Zaehringer M, Strohe D, Stuetzer H, Boecker J, Lackner K. Postcatheterization pseudoaneurysm: results of USguided percutaneous thrombin injection in 240 patients. Radiology. 2005;236:1104-10.

19. Araoz PA, Andrews JC. Direct percutaneous embolization of visceral artery aneurysms: techniques and pitfalls. J Vasc Interv
Radiol. 2000;11:1195-200.

20. Gupta V, Galwa R, Khandelwal N, Bapuraj JR. Postpyelolithotomy renal artery pseudoaneurysm management with percutaneous thrombin injection: a case report. Cardiovasc Intervent Radiol. 2008;31:422-6.

21. Han MH, Seong SO, Kim HD, Chang KH, Yeon KM, Han MC. Craniofacial arteriovenous malformation: preoperative embolization with direct puncture and injection of n-butyl cyanoacrylate. Radiology. 1999;211:661-6.

22. Lal A, Kumar A, Prakash M, Singhal M, Agarwal MM, Sarkar D, et al. Percutaneous cyanoacrylate glue injection into the renal pseudoaneurysm to control intractable hematuria after percutaneous nephrolithotomy. Cardiovasc Intervent Radiol. 2009;32:767-71.

23. Gulati GS, Gulati MS, Makharia G, Hatimota P, Saikia N, Paul $\mathrm{SB}$, et al. Percutaneous glue embolization of a visceral artery pseudoaneurysm in a case of sickle cell anemia. Cardiovasc Intervent Radiol. 2006;29:665-8.

24. Aytekin C, Firat A, Gultekin B, Boyvat F, Tasdelen A. [USguided glue injection in the treatment of femoral pseudoaneurysms]. Tani Girisim Radyol. 2003;9:257-9.

25. Rosch J, Petersen BD, Hall LD, Ivancev K. Interventional treatment of hepatic arterial and venous pathology: a commentary. Cardiovasc Intervent Radiol. 1990;13:183-8.

26. Larson RA, Solomon J, Carpenter JP. Stent graft repair of visceral artery aneurysms. J Vasc Surg. 2002;36:1260-3.

27. Won YD, Ku YM, Kim KT, Kim KH, Kim JI. Successful management of a ruptured hepatic artery pseudoaneurysm with a stent-graft. Emerg Radiol. 2009;16:247-9.

28. Mays ET, Wheeler CS. Demonstration of collateral arterial flow after interruption of hepatic arteries in man. $N$ Engl J Med. 1974;290:993-6.

29. Hoevels J, Nilsson U. Intrahepatic vascular lesions following nonsurgical percutaneous transhepatic bile duct intubation. Gastrointest Radiol. 1980;5:127-35.

30. Roche A, Doyon D, Harry G, Weingarten A, Edouard A. [Hepatic arterial embolisation. 35 cases (author's transl)]. Nouv Presse Med. 1978;7:633-7. 